\title{
GRB AFTERGLOWS: A STORY YET TO BE WRITTEN
}

\author{
S. Covino ${ }^{1}$
}

\begin{abstract}
This is brief summary of the joint discussion about GRB afterglows held in Marbella during the Gamma-Ray Burst Symposium 2012. It is based on hints proposed by many authors in their talks and offers a (personally biased) view of some of the open issues in the field. No attempts have actually been applied to really cover all the discussed subjects, and consequently only a few topics are chosen as representatives of the activities going on in the field, admittedly with some emphasis for observational results.
\end{abstract}

\section{The external shock scenario}

There are no doubts that the availability of a new generation of optical imagers as GROND $^{2}$ (Greiner et al. 2008) are providing amazing datasets able to better set several questions affecting the discussions about afterglow modeling (e.g. Greiner et al. 2011). GROND data essentially allow a subset of the optical afterglows to be studied with a richness of spectral information often comparable to that provided by the XRT aboard Swift $^{3}$. It is not therefore a surprise to discover well sampled and with high quality data events (e.g. Filgas et al. 2012) during the afterglow evolution not easily classifiable within the standard external shock scenario (Piran 2004; Zhang \& Mészáros 2004) in the optical/NIR range. This might partly question a common belief requiring that the optical afterglow is often a "bona fide" external shock emission while in the soft X-rays energy range there are contributions of multiple processes.

\section{Prompt/afterglow data correlations}

A proper coverage of the optical/NIR afterglow evolution seems mandatory for any improvements of our knowledge of the phenomenology. And it is intriguing

\footnotetext{
1 INAF / Brera Astronomical Observatory, via E. Bianchi 46, 23807, Merate (LC), Italy

${ }^{2}$ http://www.mpe.mpg.de/ jcg/GROND/

${ }^{3}$ https://heasarc.gsfc.nasa.gov/docs/swift/swiftsc.html
} 
to see that as soon as samples of events with adequate follow-up data are built new possible correlations are proposed and discussed (Oates et al. 2012) reheating a field already providing results still waiting for a proper interpretation. It was stressed that some of the observed correlations, both between afterglow parameters or between prompt and afterglow data, can more easily be interpreted in alternative scenarios as the "cannonball" model (Dado \& Dar 2012, 2013). A different approach to a statistical study of afterglow (and prompt) data is based on the selection of GRB samples highly complete in redshift (Salvaterra et al. 2012), likely allowing to derive a more reliable statistical description of GRB parameters for the events satisfying the selection criteria. In particular, the well known and highly debated "Amati/Ghirlanda/Yonetoku" correlations (Nava et al. 2012) hold for a subset of bright, at high energies, Swift GRBs.

\section{Afterglow polarization}

After the successful polarimetric campaigns carried out mainly with the ESO-VLT (Covino 2009) showing that late-time afterglows are polarized at a few per cent level, and the exciting detection of high polarization during the early afterglow (Steele et al. 2009; Uehara et al. 2012) the field is now offering new results based on intensive campaigns devoted to single events observable under favorable conditions. In particular very stringent upper limits on the circular polarization of late-time optical afterglows have been derived (Wiersema et al. 2012), allowing the possibility to put meaningful constrains on any ordered magnetic field components in the optical afterglow emitting region.

\section{Short GRBs}

Short GRBs are still attracting a considerable interest in the community in spite of their elusive nature compared to the longer durations GRBs (Kann et al. 2011; Kann 2013). Again GROND is providing a wealth of data of unprecedented quality (Nicuesa Guelbenzu et al. 2012; Rossi et al. 2012). The observationally-driven classification threshold between short and long duration GRBs at about $2 \mathrm{~s}$ has been questioned (Bromberg et al. 2013), suggesting that a fair fraction of Swift GRBs classified as short can actually be long as due to an instrument spectral sensitivity bias for the detection of generic short duration events, coupled with the intrinsic duration distribution of the two GRB classes.

\section{GRB environment}

The study of the environment of GRBs has been commonly carried out by lowand high-resolution spectroscopy (e.g. D'Elia et al. 2011; Piranomonte et al. 2011; Vergani et al. 2011) as by means of host galaxy studies (Graham \& Fruchter 2013; Perley et al. 2012; Savaglio 2013; Savaglio et al. 2012; Tanvir et al. 2012). Alternatively, information about the GRB line of sights have been derived by 
analysis of large samples of X-ray and optical/NIR observations (Campana et al. 2010, 2012).

\section{References}

Bromberg, O., Nakar, E., Piran, T., \& Sari, R., 2013 [arXiv:1210. 0068]

Campana, S., Thöne, C.C., de Ugarte Postigo, A., et al., 2010, MNRAS, 402, 2429

Campana, S., Salvaterra, R., Melandri, A., et al., 2012, MNRAS, 421, 1697

Covino, S., 2009 [arXiv:0906.5440]

Dado, S., \& Dar, A., 2012, ApJ, 749, 100

Dado, S., \& Dar, A., 2013 [arXiv: 1207.3630]

D'Elia, V., Campana, S., Covino, S., et al., 2011, MNRAS, 418, 680

Filgas, R., Greiner, J., Schady, P., et al., 2012, A\&A, 546, 101

Graham, J.F., \& Fruchter, A.S., 2013 [arXiv:1211.7068]

Greiner, J., Bornemann, W., Clemens, C., et al., 2008, PASP, 120, 405

Greiner, J., Krüler, T., Klose, S., et al., 2011, A\&A, 526, 30

Kann, D.A., Klose, S., Zhang, B., et al., 2011, ApJ, 734, 96

Kann, D.A., 2013 [arXiv: 1212.0040]

Nava, L., Salvaterra, R., Ghirlanda, G., et al., 2012, MNRAS, 421, 1256

Nicuesa Guelbenzu, A., Klose, S., Greiner, J., et al., 2012, A\&A, 548, 101

Oates, S.R., Page, M.J., De Pasquale, M., et al., 2012, MNRAS, 426, 86

Perley, D., Modjaz, M., Morgan, A.N., et al., 2012, ApJ, 758, 122

Piran, T., 2004, RvMP, 76, 1143

Piranomonte, S., Vergani, S.D., Onori, F., et al., 2011, AN, 332, 283

Rossi, A., Klose, S., Ferrero, P., et al., 2012, A\&A, 545, 77

Salvaterra, R., Campana, S., Vergani, S.D., et al., 2012, ApJ, 749, 68

Savaglio, S., 2013 [arXiv: 1212.0144]

Savaglio, S., Rau, A., Greiner, J., et al., 2012, MNRAS, 420, 627

Steele, I.A., Mundell, C.G., Smith, R.J., et al., 2009, Nature, 462, 767

Tanvir, N.R., Levan, A.J., Fruchter, A.S., et al., 2012, ApJ, 754, 46

Uehara, T., Toma, K., Kawabata, K.S., et al., 2012, ApJ, 752, 6

Vergani, S.D., Flores, H., Covino, S., et al., 2011, A\&A, 535, 127

Wiersema, K., Curran, P.A., Krüler, T., et al., 2012, MNRAS, 426, 2

Zhang, B., \& Mészáros, P., 2004, IJMPA, 19, 2385 
